\title{
A neural network model of curiosity-driven infant categorization
}

\author{
Katherine E. Twomey \\ Department of Psychology \\ Lancaster University, UK \\ Email: k.twomey@lancaster.ac.uk \\ https://sites.google.com/site/ktwomeyresearch/
}

\author{
Gert Westermann \\ Department of Psychology \\ Lancaster University, UK \\ Email: g.westermann@lancaster.ac.uk \\ http://www.psych.lancs.ac.uk/people/gert- \\ westermann
}

\begin{abstract}
Infants are curious learners who drive their own cognitive development by imposing structure on their learning environments as they explore. Understanding the mechanisms underlying this curiosity is therefore critical to our understanding of development. However, very few studies have examined the role of curiosity in infants' learning, and in particular, their categorization; what structure infants impose on their own environment and how this affects learning is therefore unclear. The results of studies in which the learning environment is structured a priori are contradictory: while some suggest that complexity optimizes learning, others suggest that minimal complexity is optimal, and still others report a Goldilocks effect by which intermediate difficulty is best. We used an autoencoder network to capture empirical data in which 10-monthold infants' categorization was supported by maximal complexity [1]. When we allowed the same model to choose stimulus sequences based on a "curiosity" metric which took into account the model's internal states as well as stimulus features, categorization was better than selection based solely on stimulus characteristics. The sequences of stimuli chosen by the model in the curiosity condition showed a Goldilocks effect with intermediate complexity. This study provides the first computational investigation of curiosity-based categorization, and points to the importance characterizing development as emerging from the relationship between the learner and its environment.
\end{abstract}

Index Terms - Curiosity, Intrinsic Motivation, Infant Categorization, Neural Network, Variability

\section{INTRODUCTION}

Infants are curious learners who explore their environment and engage selectively with stimuli based on their learning history and in-the-moment properties of the task in hand [2], [3]. Importantly, they do so largely autonomously and without feedback or extrinsic reward: infants are intrinsically motivated to learn [4]. By structuring their own learning, then, infants drive their own development. However, infants' self-generated environment is substantially different to the adult equivalent [5]. Understanding infants' curiosity-based exploration is therefore critical to our understanding of development more broadly.

Curiosity-based intrinsic motivation systems are increasingly used in the development of autonomous computational systems [6], and a growing body of computational and robotic work explores the intrinsic motivation mechanisms which subserve a range of cognitive functions and behaviors, for example low-level perceptual encoding [7], novelty detection [8], reaching [9] and motion planning [10]. Further, based on insights from developmental psychology, recent work has highlighted the value of incorporating developmental constraints in curiosity-based computational and robotic learning systems [11-13]. However, fewer studies have examined the role of curiosity from the infant's perspective, and in particular its role in the processes that underlie a fundamental component of infant cognition and development - categorization [14].

A wealth of empirical studies demonstrates that infants possess impressive categorization abilities from birth [15]. Categorization is also flexible [16] and highly sensitive to task influences such as presentation order [17] and perceptual variability [18]. These studies, which generally employ rigorously controlled stimuli to facilitate comparison between different sources of category information, have yielded mixed results. For example, Bulf, Johnson \& Valenza [19] demonstrated that newborns demonstrated learning when sequences of visual stimuli were highly predictable, but not when stimuli were less predictable. Similar phenomena have been seen in noun generalization tasks, in which older toddlers have been shown to generalize categories after training with perceptually simple - but not complex - stimuli [20]. In contrast, 10-month-old infants in a novelty preference/categorization task formed a robust category when familiarized with novel stimuli in an order that maximized, but not minimized, overall perceptual differences between successive stimuli [1]. Similarly, Quinn \& Bhatt [21] demonstrated that 6- to 7-month-old infants formed a category when successive stimuli varied between trials but not when successive stimuli were identical. Still other studies have uncovered a "Goldilocks" effect, in which learning is optimal when stimuli are of intermediate difficulty. In particular, a series of recent experiments showed that 8-month-old infants looked longer in response to auditory or visual stimuli that were moderately, but not maximally or minimally, predictable [22], [23], and older children in a hybrid word learning/categorization task retained novel label/object mappings when category exemplars were of intermediate perceptual variability [24]. Overall, then, it is not clear what degree of difficulty is optimal for infant categorization. Further, whether infants can generate this structure for 
themselves during curiosity-driven categorization is not known. Here we explore these questions in two neural network simulations of infant categorization. A first simulation is shown to capture the empirical results of Mather \& Plunkett ([1]; E1). In a second simulation we demonstrate that curiosity-based stimulus selection leads to optimal learning (E2).

\section{A NEURAL NETWORK MODEL OF THE EFFECT OF PERCEPTUAL VARIABILITY ON INFANT CATEGORIZATION}

In a preferential looking study, Mather and Plunkett ([1]; henceforth M\&P) trained 48 10-month-old children with eight exemplars of a novel animal category. Each stimulus included four features (leg length, neck length, tail thickness and ear separation; see Fig. 1) which varied systematically in magnitude across four out of five possible values. Although all children saw the same stimuli, half saw them in sequences which maximized mean overall Euclidean distance (ED) between stimuli, and half saw them in sequences which minimized that distance. At test, all children saw two simultaneously presented stimuli: one peripheral exemplar (a new exemplar with extreme feature values) and one categorycentral, prototypical stimulus (a new exemplar with the average value for each feature dimension).

It is broadly agreed that given sufficient experience with a stimulus, infants exhibit a novelty preference and look longer to novel than to familiar stimuli [25]. Based on this, in looking time experiments if infants look for longer at one test stimulus than another it is inferred that the infant has formed a category during training that excludes the preferred (i.e., novel) stimulus. In M\&P, only infants in the maximum distance condition showed evidence of having formed a category (i.e., above-chance preference for the peripheral stimulus which was more novel than the category prototype). Infants in the minimum distance condition showed no preference. The authors concluded that when successive differences between stimuli were maximized, infants were forced to traverse more of the "category space" than their peers in the minimum distance condition, leading to a stronger or more accurate category representation in the maximum distance condition.

As with any behavioral study, M\&P's data provide a snapshot of a particular age group's performance in a particular task; however, such data do not allow us to explore the cognitive mechanisms underlying these behaviors [26]. We therefore used a neural network model to simulate the effect of difficulty in M\&P's task. Closely following M\&P's design, in Experiment 1 we trained our model with stimulus sets in which mean between-stimulus Euclidean distance was maximal and minimal, respectively, and also with sequences with intermediate or random between-stimulus distances. We tested the model on novel peripheral and prototypical stimuli. Based on M\&P, we expected the model to form the most robust/accurate category after training with maximum distance stimuli, then intermediate distance, and finally minimum distance.
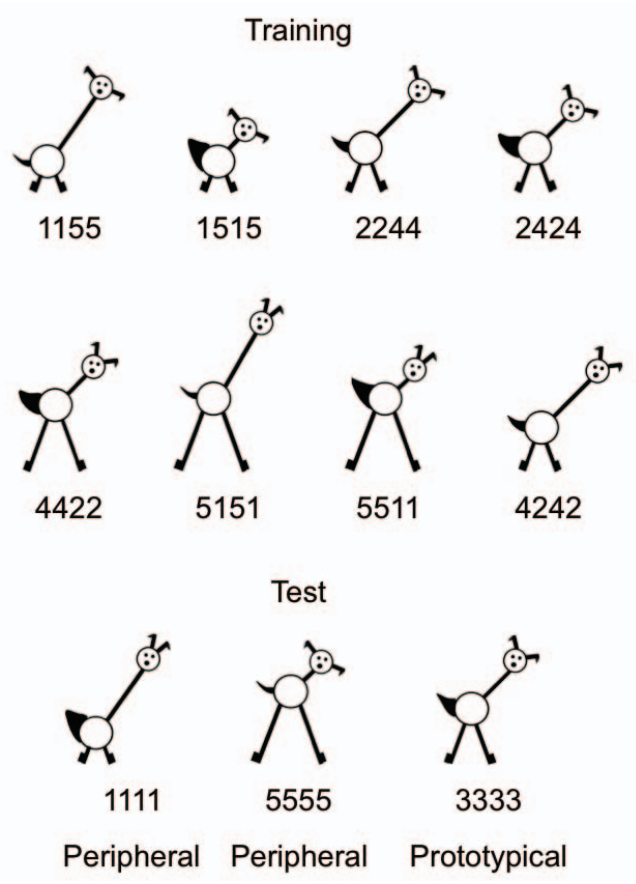

Figure 1. Stimuli used in Mather \& Plunkett [1] and the current study

\section{A. Model architecture}

We used an auto-encoder architecture that has previously captured patterns of infant categorization (e.g. [27]; Fig. 2). Auto-encoders are feed-forward connectionist neural networks consisting of an input layer, a hidden layer and an output layer. These models learn to reproduce input patterns on their output layer by comparing input and output activation after presentation of training stimuli, then adjusting the weights between units using backpropagation of this error. The current network consisted of four input units, three hidden units, and four output units. Each input unit corresponded to one of the four features of the training stimuli (i.e., leg length, neck length, tail thickness and ear separation). Hidden and output units used a sigmoidal activation function and weights were initialized randomly.

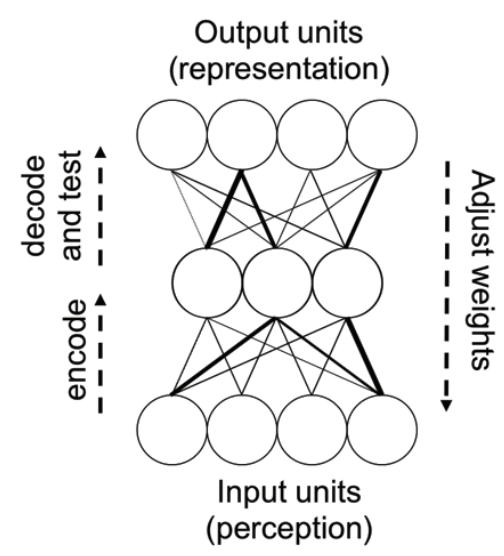

Figure 2. Model architecture 


\section{B. Stimuli}

Stimulus feature values were taken from the stimulus dimensions provided in Younger [28] (E1, Broad; see Fig. 2; these stimuli were also used by M\&P). For each feature, values were normalized to lie between 0 and 1 . Thus, features of the stimuli presented to the model had the same relative size as features in the stimuli used by M\&P.

Training sets were selected based on the mean ED between stimuli for each possible presentation order. EDs were calculated in the same way as in M\&P: all 40,320 possible presentation sequences for the eight training stimuli were created, and for each of these sequences we then calculated the mean ED between successive stimuli based on rank feature values. Stimuli for the following four conditions were selected based on mean ED:

- Maximum distance (max; cf. M\&P maximum distance): 24 sets with largest mean distance between successive stimuli

- $\quad$ Minimum distance (min; cf. M\&P minimum distance): 24 sets with smallest mean distance

- Medium distance $(m e d)$ : 24 sets with intermediate mean distance, specifically sets 20149 - 20172 when sets are sorted in order of distance (set 20160 is "middle" set)

- Random: stimuli presented in random order

The test set was identical across conditions, and consisted of two category-peripheral stimuli (new exemplars with extreme feature values) and one category-central, prototypical stimulus (new exemplar with the average value for each feature dimension).

\section{Procedure}

During training, each stimulus was presented until network error fell below threshold or for a maximum of 20 sweeps. To obtain an index of the model's learning, we tested the model with the entire training set after each sweep (with no learning) and recorded sum squared error (SSE). Order of presentation of training stimuli varied by condition (see Section B: Stimuli). We tested the model with three novel test stimuli, presented for a single sweep with no learning, and again recorded SSE. There were 24 separate models in each condition, reflecting the 24 participants in each condition of M\&P.

\section{Results}

1) Training trials. During training, infants in $M \& P$ demonstrated a significant decrease in looking from the first to the final 3-trial block. We used SSE after each training sweep as a proxy for looking time [29], [30]. For the max and min conditions we submitted SSE during the first and final 3-trial block to a 2 (block: first, last; within-subjects) $\mathrm{x}$ 2 (condition: max, min; between-subjects) mixed ANOVA ${ }^{1}$. In line with M\&P, a main effect of block $(F(1,46)=97.34$,

\footnotetext{
${ }^{1}$ A mixed effects model produced the same overall pattern of results, however due to non-convergence during model comparisons we report the ANOVA here.
}

$\left.p<.0001, \eta_{G}^{2}=.46\right)$ confirmed that overall SSE decreased from the first block $(M=0.57, S D=0.11)$ to the final block $(M=0.54, S D=0.11)$. A main effect of condition $(F(1,46)$ $\left.=2079.12, p<.0001, \eta_{G}{ }_{G}=.96\right)$ revealed that there was less error overall in the max condition $(M=0.45, S D=0.03)$ than in the min condition $(M=0.66, S D=0.03)$. Finally, there was a significant block-by-condition interaction $(F(1$, 46) $\left.=4.40, p=.041, \eta_{G}^{2}=.03\right)$, which arose from a greater decrease in SSE in the max condition (mean decrease = 0.045 ) than in the min condition (mean decrease $=0.030$ ). Thus, as with the infants in M\&P, "looking" in the model decreased over training. On the assumption that infants look away from stimuli they have fully encoded, the model predicts more robust familiarization in the max condition than the min condition, as indexed by less looking. This effect did not reach significance in M\&P's analyses. Note, however, that our model data are substantially less variable than M\&P's empirical data, likely accounting for our detection of significant differences where M\&P found null effects.

2) Test trials. In empirical categorization/novelty preference tasks, increased looking to peripheral stimuli at test is taken as evidence that infants have learned a category which includes the prototypical stimulus. Again using SSE as a proxy for looking time, we collapsed our analyses across the two peripheral stimuli [1], and calculated proportion of total test SSE to the peripheral stimulus, as depicted in Figure 3. Wilcoxon rank-sum tests ${ }^{2}$ against chance confirmed that in all conditions the model formed a category (all $V \mathrm{~s}=300$, all $p$ s <.001). However, a Kruskal-Wallis test revealed that SSE (and therefore categorization) differed between conditions $(W(3)=80.13, p<.001)$. Post-hoc, two-tailed Wilcoxon tests (Bonferroni-corrected) confirmed that the model produced more SSE in the max condition $(M d n=$ $0.99)$ than in the min condition $(M d n=0.76 ; W=576, p<$ $.0001, r=-1.53)$, the med condition ( $M d n=0.79 ; W=576$, $p<.0001, r=-1.53)$ or the random condition $(M d n=0.83$; $W=575, p<.0001, r=-1.51)$. All other between-condition differences were also significant (all $p \mathrm{~s}<.0001$ ). Note that although infants did not show evidence of category formation in M\&P's minimum distance condition, the authors argue that these infants were in fact categorizing, but not sufficiently robustly for this to be detected at test. Overall, then, the model captures the looking behavior exhibited by infants in M\&P: the model formed a more robust category after training with sequences which maximized rather than minimized overall EDs between stimuli.

\section{E. Discussion}

In Experiment 1 (as in M\&P) the order of stimulus presentation was fixed to control the mean successive ED between conditions. This created an artificially-structured environment in which the model learned best from the inputs with the most inter-stimulus variation. As noted, however, outside the lab infants are curious explorers, constructing their own infant-appropriate learning environments [5]. A

\footnotetext{
${ }^{2}$ We report non-parametric tests due to non-normality of test SSE
} 
handful of studies suggest that when they do so, there is evidence of a Goldilocks effect, where optimal learning arises from stimuli that are complex enough to provide novel information, but not so difficult that learning becomes impossible. Importantly, "complexity" depends not just on stimulus features (e.g., shape) but on the infant's own learning history [22], [23], [31]. Thus, in Experiment 2 we allowed the model to be curious; that is, to choose its own stimuli based both on environmental (i.e., ED) and intrinsic (i.e., internal states) factors.

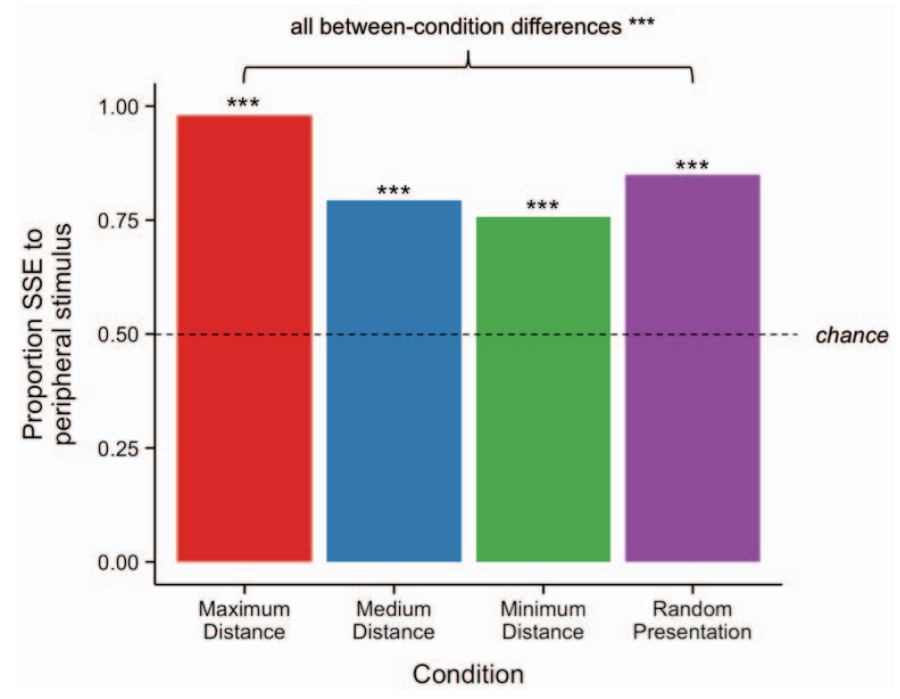

Figure 3. Proportion SSE to peripheral stimulus at test in Experiment 1. $* * * p<.001$.

\section{EXPERIMENT 2: SIMULATING CURIOSITY-DRIVEN INFANT CATEGORIZATION}

In Experiment 2, the model itself selected the order in which it received stimuli. On each training trial, it calculated a selection criterion for each possible successive stimulus, and selected the stimulus for which the absolute value of this metric was maximal.

\section{F. Model architecture}

Model architecture and parameters were identical to those used in Experiment 1.

\section{G. Procedure.}

The procedure used in Experiment 2 was identical to that used in Experiment 1, with the exception that stimulus order was determined by the model (see Section H).

\section{H. Stimuli}

Individual training and test stimuli were identical to those used in Experiment 1. However, we did not present the model with fixed stimulus orders. Instead, it chose the sequence in which it saw stimuli, with the constraint that each stimulus was seen exactly once.

We provided the model with two methods of stimulus selection:
- $\quad$ Curiosity-based selection: Before presentation of each stimulus, the model calculated $(t-o) o(1-o)$ (curiosity function) for all possible successive stimuli where $t=$ possible target values and $o=$ possible output values. For example, after presentation of the first stimulus, each of the seven remaining stimuli was presented to the model for a single sweep (with no learning) and the resulting target and output values used to calculate curiosity. The next stimulus used to train the model was that for which the curiosity function was maximal.

In the curiosity function, $t-o$ represents the error in response to a given stimulus, and reflects the relationship between the model (or learner) and its targets (or the learning environment). $o(1-o)$ relates to the model's internal state, and more specifically, its plasticity: the term, which is the derivative of the sigmoid function and is part of the backpropagation weight update function, is maximal for output values of 0.5 and minimal for outputs of 0 or 1 . Therefore it defines changes in plasticity as a function of the model's output activation. Thus, our index of curiosity took into account the variability of stimuli available in the environment, but critically also incorporated aspects of the learner's internal state, allowing prior experience to affect in-the-moment learning.

- Euclidean selection: While the max condition of Experiment 1 maximized mean Euclidean distance between all stimuli in a set, the Euclidean selection condition allowed the model to select its own stimuli by calculating the Euclidean distance between the previous and all potential successive stimuli on each training trial, and selecting the stimulus for which this value was maximal.

Unlike the curiosity condition, in which the model selected its first stimulus by calculating the curiosity function based on its initial state, in the Euclidean selection condition the model compared two stimuli to calculate the selection criterion. The model's first stimulus was therefore random, and the model selected all successive stimuli.

\section{Results and discussion}

Proportion of total SSE to test trials is depicted in Figure 4. Wilcoxon rank-sum tests against chance $(0.5)$ confirmed that the model formed a category in all conditions (both $p \mathrm{~s}<$ .001). Allowing the model to choose its own stimuli therefore led to category formation irrespective of how the model made that choice. A Wilcoxon test revealed that the model generated more SSE in response to the peripheral stimulus in the curiosity condition $(M d n=0.97)$ than in the Euclidean selection condition $(M d n=0.91 ; W=495, p<$ $.0001, r=-0.92)$. Thus, the model formed a stronger category representation in the curiosity than the Euclidean condition. Importantly, this result demonstrates that using a selection criterion that takes into account the model's own internal state as well as aspects of the environment leads to better performance than a selection criterion based on environmental characteristics alone.

Interestingly, there was no difference in SSE between the curiosity and max conditions $(W=235, p=0.28, r=-0.22)$. This result raises the question if the curiosity mechanism selects the maximally novel next stimulus at each point in 
training. This would be in contrast to previous research which suggests that intermediate novelty is preferred by autonomous learners [22], [23], [31], [32]. To explore this possibility, we analyzed the stimulus sequences chosen by the model in the curiosity and Euclidean selection conditions.

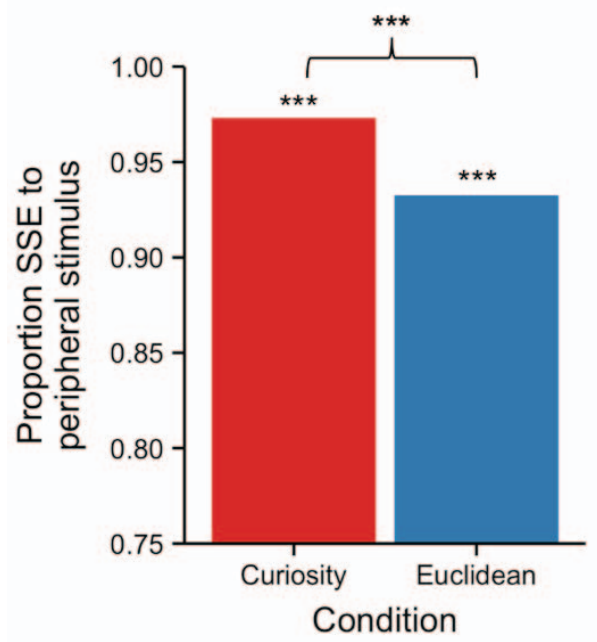

Figure 4. Proportion SSE to peripheral stimulus at test in Experiment 2. $* * * p .001$.

The entire set of 43,200 permutations of the eight training stimuli generated 281 unique overall mean EDs. Table 1 provides the rank mean ED (higher rank = greater ED) for each sequence chosen by each of the 24 models in the two conditions.

Table 1. Rank mean Euclidean Distances for the sequences chosen in the Euclidean selection and curiosity conditions

\begin{tabular}{lcc} 
Condition & Rank mean ED & Frequency/24 \\
\hline Euclidean & $2 / 281$ & 9 \\
& $14 / 281$ & 15 \\
Curiosity & $34 / 281$ & 5 \\
& $41 / 281$ & 18 \\
& $50 / 281$ & 1
\end{tabular}

Unlike in the max condition of Experiment 1, neither curiosity-based nor Euclidean selection maximized overall mean Euclidean distance. Critically, in the curiosity condition the model chose stimulus sequences with lower mean distances than in the Euclidean selection condition; in fact, analysis of the individual stimulus-by-stimulus differences revealed that the model failed to maximize successive ED for $27 \%$ of its choices. For 18 out of 24 of its sequences it first maximized and then immediately minimized ED, suggesting that curiosity-based selection maximizes learning whilst minimizing interference from successive stimuli. Thus, irrespective of whether difference in the current study is thought of in terms of mean or successive ED, the model optimized its learning by choosing stimuli of intermediate differences in the curiosity condition based on its internal states and relationship to the learning environment.

\section{General Discussion}

In Experiment 1 we used an auto-encoder to capture empirical data presented by Mather \& Plunkett [1], in which 10-monthold infants formed a robust category when familiarized with stimuli in sequences which maximized the mean perceptual distance between stimuli, but not sequences which minimized it. To explore whether curiosity-based stimulus selection would lead to similarly successful categorization, in Experiment 2 we allowed the model to choose its own stimuli based on maximizing an index of curiosity that took into account the model's internal states as well as perceptual variability, or based on maximizing perceptual differences alone. Learning, as evidenced by the strength of the resulting category, was best after curiosity-based stimulus selection, with no difference between optimum learning in Experiment 1 and Experiment 2.

These simulations raise three important questions and make strong predictions for future empirical work. First, although the model showed a Goldilocks effect in the curiosity condition, why did it choose sequences closer to the most difficult end of the spectrum, rather than sequences of true intermediate difficulty (i.e., ranked around 140/281 as indexed by mean ED)? This result depends critically on the inclusion of the output term in the curiosity function, the calculation of which incorporates previous learning as well as in-the-moment environmental input and task structure. In effect, curiositybased learning allows previous experience to affect "difficulty"; that is, intermediate difficulty is relative to the state of the learner at any given point in development. In line with dynamic systems accounts of development [33], then, intask knowledge (i.e., what level of difficulty will be optimal) is not based on a straightforward, linear relationship between features in the environment, but rather emerges from a nonlinear function of those features and the model's learning history.

Second, why was learning in the max condition, in which stimuli were chosen a priori based on environmental features, as strong as in the curiosity condition? As in M\&P, we maximized overall $\mathrm{ED}$ in this condition, resulting in sequences in which successive EDs were sometimes not maximized. The (overall) max sequences therefore provide a similar intermediate-difficulty learning environment as in the curiosity condition. Importantly, however, the max condition reflects a situation in which learning opportunities are precisely determined before learning takes place - a situation unlikely to be found outside the highly-structured lab environment. In contrast, the current model predicts that when infants are allowed to direct their own learning opportunities during categorization, their looking behavior will exhibit a Goldilocks-type effect in which optimal learning emerges from intermediate difficulty.

Finally, and perhaps most importantly, what does this model suggest about the mechanisms underlying infant categorization? The sequences chosen by the model in the curiosity condition predict that in order to prevent interference during learning, infants will initially select maximally novel 
stimuli, followed by minimally novel stimuli. Data collection exploring these two predictions with 10-month-old infants in a categorization task is currently underway. Overall, however, the current simulations unite work in in developmental psychology with recent insights from robotics and autonomous systems in the first neural network investigation of curiositydriven infant categorization. They highlight that development does not happen in isolation, due to intrinsic or extrinsic factors alone. Rather, the current studies illustrate development as a system which emerges from the dynamic interaction between the environment and learner.

\section{ACKNOWLEDGMENT}

We gratefully acknowledge the support of the ESRC International Centre for Language and Communicative Development (LuCiD; ES/L008955/1).

\section{REFERENCES}

[1] E. Mather and K. Plunkett, "Same items, different order: Effects of temporal variability on infant categorization," Cognition, vol. 119, no. 3, pp. 438-447, 2011.

[2] A. F. Pereira, L. B. Smith, and C. Yu, "A bottom-up view of toddler word learning," Psychon. Bull. Rev., vol. 21, no. 1, pp. 178-185, 2014.

[3] C. Yu and L. B. Smith, "Embodied attention and word learning by toddlers," Cognition, vol. 125, no. 2, pp. 244-262, 2012.

[4] E. Mather, "Novelty, attention, and challenges for developmental psychology," Front. Psychol., vol. 4, Aug. 2013.

[5] L. B. Smith, C. Yu, and A. F. Pereira, "Not your mother's view: The dynamics of toddler visual experience," Dev. Sci., vol. 14, no. 1, pp. 9-17, 2011.

[6] G. Baldassarre, T. Stafford, M. Mirolli, P. Redgrave, R. M. Ryan, and A. Barto, "Intrinsic motivations and open-ended development in animals, humans, and robots: an overview," Cogn. Sci., vol. 5, p. 985, 2014.

[7] L. Lonini, S. Forestier, C. Teulière, Y. Zhao, B. E. Shi, and J. Triesch, "Robust active binocular vision through intrinsically motivated learning," Front. Neurorobotics, vol. 7, p. 20, 2013.

[8] S. Marsland, U. Nehmzow, and J. Shapiro, "On-line novelty detection for autonomous mobile robots," Robot. Auton. Syst., vol. 51, no. 2, pp. 191-206, 2005.

[9] A. Shah and K. N. Gurney, "Emergent structured transition from variation to repetition in a biologically-plausible model of learning in basal ganglia," Front. Psychol., vol. 5, 2014.

[10] M. Frank, J. Leitner, M. Stollenga, A. Förster, and J. Schmidhuber, "Curiosity driven reinforcement learning for motion planning on humanoids," Front. Neurorobotics, vol. 7, p. 25, 2014.

[11] P.-Y. Oudeyer, A. Baranes, and F. Kaplan, "Intrinsically motivated learning of real-world sensorimotor skills with developmental constraints," in Intrinsically Motivated Learning in Natural and Artificial Systems, Springer, 2013, pp. 303-365.

[12] P.-Y. Oudeyer, F. Kaplan, and V. V. Hafner, "Intrinsic motivation systems for autonomous mental development," Evol. Comput. IEEE Trans. On, vol. 11, no. 2, pp. 265-286, 2007.

[13] M. Schlesinger and D. Amso, "Image free-viewing as intrinsicallymotivated exploration: estimating the learnability of center-of-gaze image samples in infants and adults," Front. Psychol., vol. 4, 2013.

[14] P. C. Quinn, P. D. Eimas, and S. L. Rosenkrantz, "Evidence for representations of perceptually similar natural categories by 3-monthold and 4-month-old infants," Perception, vol. 22, no. 4, pp. 463-475, 1993.

[15] P. C. Quinn, A. M. Slater, E. Brown, and R. A. Hayes, "Developmental change in form categorization in early infancy," $\mathrm{Br}$. J. Dev. Psychol., vol. 19, no. 2, pp. 207-218, 2001.

[16] J. S. Horst, L. M. Oakes, and K. L. Madole, "What does it look like and what can it do? Category structure influences how infants categorize," Child Dev, vol. 76, no. 3, pp. 614-631, 2005.
[17] L. M. Oakes, J. M. Plumert, J. M. Lansink, and J. D. Merryman, "Evidence for task-dependent categorization in infancy," Infant Behav Dev, vol. 19 , no. 4, pp. 425-440, 1996.

[18] K. A. Kovack-Lesh and L. M. Oakes, "Hold your horses: How exposure to different items influences infant categorization," J. Exp. Child Psychol., vol. 98, no. 2, pp. 69-93, 2007.

[19] H. Bulf, S. P. Johnson, and E. Valenza, "Visual statistical learning in the newborn infant," Cognition, vol. 121, no. 1, pp. 127-132, 2011.

[20] J. Y. Son, L. B. Smith, and R. L. Goldstone, "Simplicity and generalization: Short-cutting abstraction in children's object categorizations," Cognition, vol. 108, no. 3, pp. 626-638, 2008.

[21] P. C. Quinn and R. S. Bhatt, "Learning perceptual organization in infancy: The effect of simultaneous versus sequential variability experience," Perception, vol. 39, no. 6, p. 795, 2010.

[22] C. Kidd, S. T. Piantadosi, and R. N. Aslin, "The Goldilocks Effect: Human Infants Allocate Attention to Visual Sequences That Are Neither Too Simple Nor Too Complex," PLoS ONE, vol. 7, no. 5, p. e36399, May 2012.

[23] C. Kidd, S. T. Piantadosi, and R. N. Aslin, "The Goldilocks effect in infant auditory attention," Child Dev., vol. 85, no. 5, pp. 1795-1804, 2014.

[24] K. E. Twomey, S. L. Ranson, and J. S. Horst, "That's more like it: Multiple exemplars facilitate word learning," Infant Child Dev., vol. 23, no. 2, pp. 105-122, 2014.

[25] C. Houston-Price and S. Nakai, "Distinguishing novelty and familiarity effects in infant preference procedures," Infant Child Dev., vol. 13, no. 4, pp. 341-348, 2004.

[26] G. Westermann and D. Mareschal, "Mechanisms of developmental change in infant categorization," Cogn. Dev., vol. 27, no. 4, pp. 367$382,2012$.

[27] R. M. French, D. Mareschal, M. Mermillod, and P. C. Quinn, "The role of bottom-up processing in perceptual categorization by 3-to 4month-old infants: Simulations and data," J. Exp. Psychol.-Gen., vol. 133, no. 3, pp. 382-397, 2004.

[28] B. A. Younger, "The segregation of items into categories by tenmonth-old infants," Child Dev., pp. 1574-1583, 1985.

[29] D. Mareschal and R. French, "Mechanisms of Categorization in Infancy," Infancy, vol. 1, no. 1, pp. 59-76, 2000.

[30] G. Westermann and D. Mareschal, "From perceptual to languagemediated categorization," Philos. Trans. R. Soc. B Biol. Sci., vol. 369, no. 1634, p. 20120391, 2014

[31] J. Schmidhuber, "Maximizing fun by creating data with easily reducible subjective complexity," in Intrinsically Motivated Learning in Natural and Artificial Systems, Springer, 2013, pp. 95-128.

[32] D. E. Berlyne, Conflict, arousal, and curiosity. New York, NY: McGraw-Hill Book Company, 1960.

[33] E. Thelen and L. B. Smith, A Dynamic Systems Approach to the Development of Cognition and Action. Cambridge, Mass.: MIT Press, 1996. 